\title{
Impact of pulmonary interstitial lesions on efficacy and prognosis of EGFR-TKI-treated advanced non-small cell lung cancers
}

\author{
Xiang-Yan Zhang ${ }^{1}$, Rui Cao ${ }^{1}$, Yi-Jia Guo ${ }^{1}$, Yan-Hua Zhen ${ }^{2}$, Jia-He Zheng' ${ }^{2}$, Le-Tian Huang ${ }^{1}$, \\ Shu-Ling Zhang ${ }^{1}$, Wei Jing ${ }^{1}$, Li Sun ${ }^{1}$, Jian-Zhu Zhao ${ }^{1}$, Cheng-Bo Han ${ }^{1}$, Jie-Tao Ma ${ }^{1}$ \\ ${ }^{1}$ Department of Clinical Oncology, ${ }^{2}$ Department of Radiology, Shengjing Hospital of China Medical University, Shenyang 110022, China \\ Contributions: (I) Conception and design: XY Zhang, JH Zheng, CB Han, JT Ma; (II) Administrative support: JH Zheng, CB Han, JT Ma; (III) \\ Provision of study materials or patients: JH Zheng, LT Huang, SL Zhang, W Jing, L Sun, JZ Zhao, CB Han, JT Ma; (IV) Collection and assembly \\ of data: XY Zhang, R Cao, YJ Guo, YH Zhen; (V) Data analysis and interpretation: XY Zhang, SL Zhang, W Jing, JT Ma; (VI) Manuscript writing: \\ All authors; (VII) Final approval of manuscript: All authors. \\ Correspondence to: Jie-Tao Ma, MD, PhD. Department of Clinical Oncology, Shengjing Hospital of China Medical University, Shenyang 110022, \\ China. Email: majt@sj-hospital.org.
}

Background: This study aimed to assess the impact of pre-existing pulmonary interstitial lesions (PIL) on the efficacy and prognosis of patients with epidermal growth factor receptor (EGFR) mutant non-small cell lung cancer (NSCLC) treated with EGFR tyrosine kinase inhibitor (TKI).

Methods: Patients with advanced NSCLC harboring EGFR exon 19 deletion (E19 del) or exon 21 (E21) L858R were enrolled in this study. All patients underwent high resolution computed tomography (HRCT) chest scans prior to EGFR-TKI treatment. Pre-existing PIL was graded according to HRCT imaging (PIL 0,1,2, and 3). Cox proportional-hazards regression models were used to identify the prognostic factors for progression-free survival (PFS).

Results: A total of 134 eligible patients were enrolled. The overall objective response rate (ORR) and median PFS were $73.1 \%$ and 10.0 months (95\% CI: 7.51-12.49), respectively. There were 62 (46.3\%), 25 (18.7\%), 28 (20.9\%), and 19 (14.1\%) cases of PIL grade 0, 1, 2, and 3, respectively, with median PFS and ORR of 12.9 months and 80.6\%, 11.0 months and $72.0 \%, 10.0$ months and $71.4 \%$, and 7.0 months and $52.6 \%$, respectively. Multivariate analysis showed that squamous cell carcinoma (vs. adenocarcinoma, HR =4.33), E21 L858R (vs. E19 del, HR =1.57), and PIL grade 3 (vs. grade 0-2, HR =1.60-2.48) were poor prognostic factors for PFS $(\mathrm{P}<0.05$ for all).

Conclusions: Pre-existing PIL grade is an independent prognostic factor for predicting resistance to EGFR-TKIs in patients with EGFR-mutant advanced NSCLC. Higher PIL grade suggests higher risk of early progression.

Keywords: Epidermal growth factor receptor (EGFR); non-small cell lung cancer (NSCLC); prognosis; pulmonary interstitial lesions (PIL); tyrosine kinase inhibitors (TKIs)

Submitted Aug 11, 2019. Accepted for publication Dec 10, 2019.

doi: $10.21037 /$ jtd.2019.12.128

View this article at: http://dx.doi.org/10.21037/jtd.2019.12.128

\section{Introduction}

Lung cancer is the leading cause of cancer death worldwide. Non-small cell lung cancer (NSCLC) accounts for $85 \%$ of lung cancer cases (1). For advanced NSCLC with epidermal growth factor receptor (EGFR) mutation, EGFR tyrosine kinase inhibitors (EGFR-TKIs) serve as standard first-line therapies with a response rate of over $70 \%$ and a median progression-free survival (PFS) of 9-13 months $(2,3)$. However, patients receiving the EGFR-TKI treatment almost inevitably develop disease progression due to primary or secondary drug resistance. The mechanism of 
resistance is complicated, with EGFR T790M accounting for approximately $50 \%$ of its emergence. Mesenchymalepithelial transition amplification, epithelial-mesenchymal transition (EMT), and small cell transformation can also lead to drug resistance (4). Once tumor cells acquire the properties of EMT, their invasive ability and metastasis will be enhanced, thereby resulting in drug resistance (5).

EMT is also a mode of interstitial cell production in the process of pulmonary fibrosis and is significantly related to interstitial lung disease (ILD) (6). TGF- $\beta$ is a fibrogenic cytokine that can induce EMT and is closely related to pulmonary fibrosis (7-9). Furthermore, previous studies have shown that ILD is closely related to carcinogenesis in lung cancer and that the incidence of ILD upon lung cancer diagnosis varied between 2.4-10.9\% (10-12). ILD patients with lung cancer had a poor prognosis (10).

Based on the relationship of EMT with pulmonary fibrosis and EGFR-TKI resistance, it is worth investigating whether there is a connection between pulmonary fibrosis and EGFR-TKI resistance. Therefore, this study evaluated the effect of pre-existing pulmonary interstitial lesions (PIL) based on high-resolution computed tomography (HRCT) imaging on the efficacy and prognosis of EGFRTKIs for advanced EGFR-mutant NSCLC and explored the relationship between the extent of PIL and EGFR-TKI resistance.

\section{Methods}

\section{Patient selection}

We retrospectively reviewed patient records at the Cancer Center in the Shengjing Hospital of China Medical University between January 2016 and April 2018. Patients who met the following inclusion criteria were enrolled: (I) histologically or cytologically confirmed NSCLC; (II) recurrence or unresectable stage III or IV, according to the seventh edition of the tumor-node-metastasis (TNM) classification for lung cancer by the American Joint Committee on Cancer; (III) EGFR exon 19 deletion (E19 del) or exon 21 (E21) L858R mutation; (IV) received first-generation EGFR-TKI monotherapy; (V) HRCT imaging (256-section CT scanner, Brilliance iCT ${ }^{\circledR}$; Philips Healthcare, Cleveland, OH, USA) and follow-up information before and after EGFR-TKI treatment was available. Patients were excluded if they had one of the following exclusion criteria: (I) received chemotherapy or other targeted therapy during EGFR-TKI treatment;
(II) EGFR-TKIs were only used as consolidation or maintenance therapy; (III) harbored rare or co-mutated EGFR mutations; (IV) previously received EGFR-TKI treatment; (V) second- or third-generation EGFR-TKIs; (VI) had a history of chest radiotherapy before or during EGFR-TKI treatment.

Eligible patients' clinicopathological characteristics were collected and included age, sex, smoking history, Eastern Cooperative Oncology Group performance status (ECOG PS), Charlson comorbidity index (CCI), pathological type, clinical stage, EGFR mutation type, EGFR-TKI category, PIL grade, and follow-up information. Treatment response was evaluated according to the Response Evaluation Criteria in Solid Tumors 1.1. PFS was defined as the time in months from the initiation of EGFR-TKI treatment to disease progression.

\section{Grading criteria for PIL}

HRCT imaging PIL features included ground-glass opacity, interlobular septal thickening, interstitial hyperplasia, honeycomb sign, reticulation, consolidation, patchy shadow, tractive bronchiectasis, and bullae $(13,14)$. Referring to the HRCT features for usual interstitial pneumonia in the ATS/ ERS/JRS/ALAT International Guidelines and previous studies of ILD (14-16), grading criteria for PIL based on chest HRCT imaging were proposed (Table 1 and Figure 1). Each patient's PIL grade was independently verified by two chest radiologists, according to the chest HRCT before the EGFR-TKI treatment. In cases of disagreement between radiologists, another radiologist was consulted until a consensus was reached.

\section{Statistical analysis}

Statistical analysis was performed using the SPSS statistical software version 21.0 (IBM Corporation, Armonk, NY, USA). In this study, continuous variables were statistically analyzed using the median binary classification. A chisquared test was used for categorical variable correlation analysis. Median PFS was estimated using the KaplanMeier method, where curves were compared using the logrank test. Potential prognostic factors were identified by univariate analysis, where $\mathrm{P}<0.1$ was considered statistically significant. Multivariable Cox proportional hazard models were used to calculate adjusted hazard ratios (HRs) and their $95 \%$ confidence intervals $(\mathrm{CIs})$, where $\mathrm{P}<0.05$ was considered statistically significant. 
Table 1 PIL grading criteria based on HRCT imaging

\begin{tabular}{ll}
\hline PIL grade & Description \\
\hline Grade 0 (no interstitial change) & No signs of interstitial lesions \\
Grade 1 (mild interstitial change) & $\begin{array}{l}\text { Ground glass opacification, focal or non-segmental distribution, without reticular } \\
\text { abnormalities, honeycombing and traction bronchiectasis }\end{array}$ \\
Grade 2 (moderate interstitial change) & $\begin{array}{l}\text { Reticular abnormalities in one or two lobes, without honeycombing and traction } \\
\text { bronchiectasis }\end{array}$ \\
Grade 3 (severe interstitial change) & $\begin{array}{l}\text { Reticular abnormalities in more than two lobes, and/or honeycombing with or without } \\
\text { traction bronchiectasis }\end{array}$ \\
\hline
\end{tabular}

PIL, pulmonary interstitial lesions; HRCT, high resolution computed tomography.
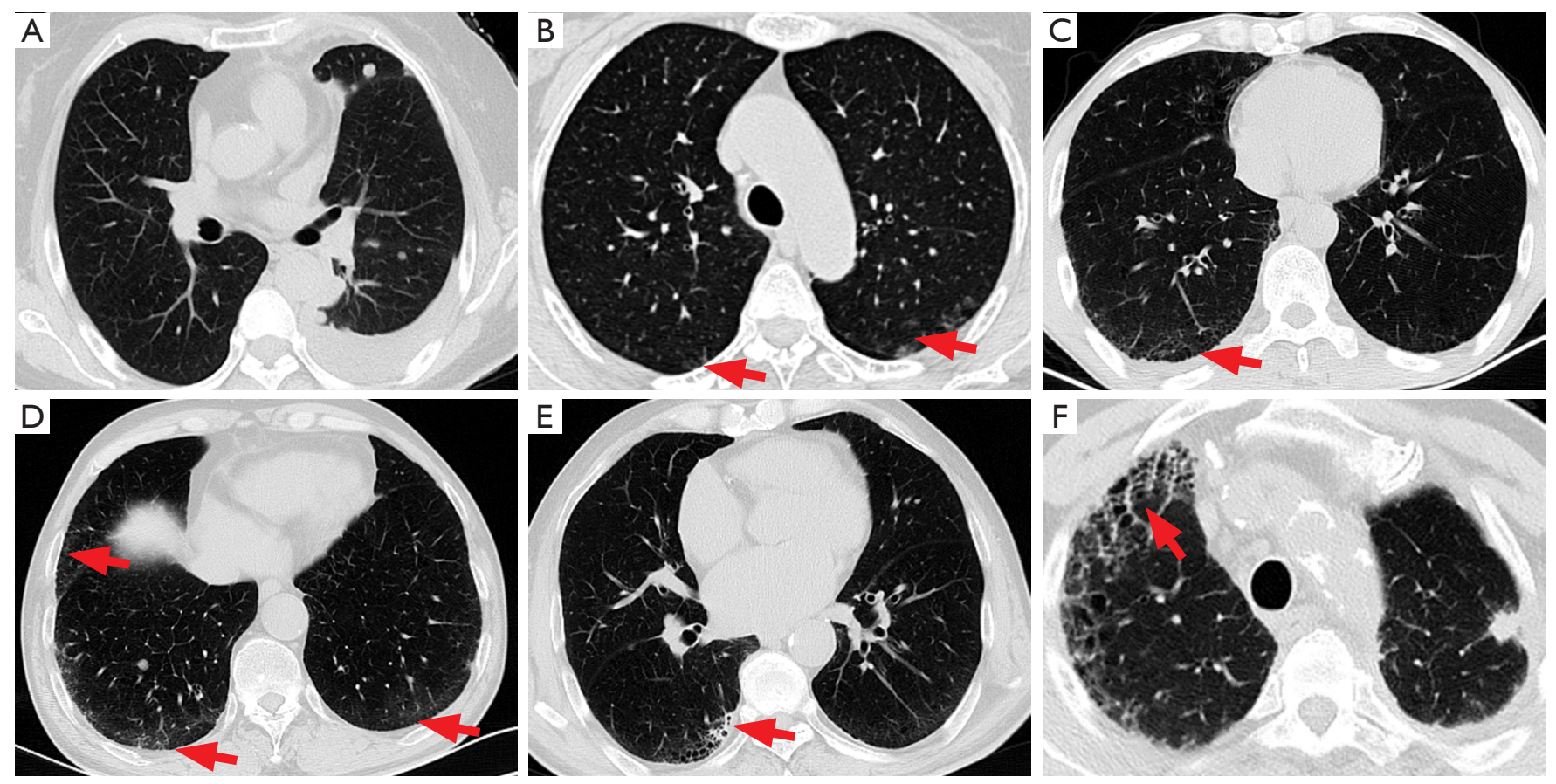

Figure 1 Grading criteria for PIL based on chest HRCT imaging. (A) Grade 0: no interstitial lung abnormalities; (B) grade 1: scattered ground-glass opacification in bilateral upper lobes; (C) grade 2: reticulation changes in right lower lobe; (D) grade 3: reticular abnormalities in three lobes (right middle and bilateral lower lobes) and (E) honeycombing in right lower lobe; and (F) grade 3: honeycombing with traction bronchiectasis in right upper lobe. PIL, pulmonary interstitial lesions; HRCT, high resolution computed tomography.

\section{Results}

\section{Patient characteristics}

Patient characteristics are summarized in Table 2. A total of 134 eligible patients were enrolled in this study. The median age was 61 years (range, 37-86 years), 85 patients were female (63.4\%), 101 patients had ECOG PS 1 (75.4\%), 129 patients had adenocarcinoma (96.3\%), 128 patients had stage IV disease (95.6\%), 58 patients had EGFR E19 del (43.3\%), 29 patients had surgical history of lung cancer (21.6\%), and 94 patients had first-line treatment (70.1\%).

\section{PIL grade and correlation analysis}

According to the PIL grading criteria based on CT imaging, there were 62 patients with PIL grade 0 (46.3\%), 25 patients with PIL grade 1 (18.7\%), 28 patients with PIL grade 2 (20.9\%), and 19 patients with PIL grade 3 (14.1\%, 
Table 2 Clinicopathological characteristics of the included patients

\begin{tabular}{|c|c|}
\hline Characteristics & Number (\%) \\
\hline \multicolumn{2}{|l|}{ Age } \\
\hline$\leq 60$ & $65(48.5)$ \\
\hline$>60$ & $69(51.5)$ \\
\hline \multicolumn{2}{|l|}{ Gender } \\
\hline Male & $49(36.6)$ \\
\hline Female & $85(63.4)$ \\
\hline \multicolumn{2}{|l|}{ Smoking } \\
\hline Never & $106(79.1)$ \\
\hline Current/former & $28(20.9)$ \\
\hline \multicolumn{2}{|l|}{ ECOG PS } \\
\hline 1 & $101(75.4)$ \\
\hline 2 & $20(14.9)$ \\
\hline 3 & $13(9.7)$ \\
\hline \multicolumn{2}{|l|}{$\mathrm{CCl}$ score } \\
\hline$\leq 8$ & $101(75.4)$ \\
\hline$>8$ & $33(24.6)$ \\
\hline \multicolumn{2}{|l|}{ Pathological type } \\
\hline Adenocarcinoma & $129(96.3)$ \\
\hline Squamous cell carcinoma & $5(3.7)$ \\
\hline \multicolumn{2}{|l|}{ EGFR mutation type } \\
\hline E19 del & $58(43.3)$ \\
\hline E21 L858R & $76(56.7)$ \\
\hline \multicolumn{2}{|l|}{ TNM stage (AJCC 7th) } \\
\hline IIIA & $3(2.2)$ \\
\hline IIIB & $3(2.2)$ \\
\hline IVA & $46(34.4)$ \\
\hline IVB & $82(61.2)$ \\
\hline \multicolumn{2}{|l|}{ History of chemotherapy } \\
\hline No & $75(54.6)$ \\
\hline Yes & $59(45.4)$ \\
\hline \multicolumn{2}{|l|}{ Surgical history of lung } \\
\hline No & $105(78.4)$ \\
\hline Yes & $29(21.6)$ \\
\hline
\end{tabular}

Table 2 (continued)
Table 2 (continued)

\begin{tabular}{ll}
\hline Characteristics & Number $(\%)$ \\
\hline EGFR-TKI category & $66(49.3)$ \\
Gefitinib & $31(23.1)$ \\
Icotinib & $37(27.6)$ \\
Erlotinib & \\
Treatment line & $94(70.1)$ \\
First-line & $40(19.9)$ \\
$\geq$ Second-line & \\
PIL grade & $62(46.3)$ \\
PIL0 & $25(18.7)$ \\
PIL1 & $28(20.9)$ \\
PIL2 & $19(14.1)$ \\
PIL3
\end{tabular}

EGFR-TKI, epidermal growth factor receptor-tyrosine kinase inhibitor; PIL, pulmonary interstitial lesions; ECOG PS, Eastern Cooperative Oncology Group performance status; $\mathrm{CCl}$, Charlson comorbidity index; TNM, tumor-node-metastasis.

Table 2). Correlation analysis between PIL grade and other variables showed that only surgical history of lung cancer was weakly correlated with PIL grade, while age, sex, smoking history, CCI, EGFR mutation type, pathological type, and clinical stage were not related to PIL grade (Table 3).

\section{Independent risk factors for disease progression}

The objective response rate (ORR) and median PFS of the patients receiving EGFR-TKI treatment were $73.1 \%$ and 10.0 months (95\% CI: 7.51-12.49), respectively. PFS rates for 1,2 , and 3 years were $45 \%, 20 \%$, and $5 \%$, respectively. Univariate analysis showed that sex, age, clinical stage, smoking history, ECOG PS, CCI, EGFR-TKI category, history of chemotherapy, and treatment lines were not significantly associated with PFS ( $\mathrm{P}>0.1$ for all). In turn, pathological type, EGFR mutation type, surgical history of lung cancer, and PIL grade were associated with PFS $(\mathrm{P}<0.1$ for all $)$.

Multivariate analysis showed that there was no significant association between surgical history of lung cancer and PFS, while pathological type, EGFR mutation type, and 
Table 3 The correlations between pulmonary interstitial lesions (PIL) and other clinicopathological factors

\begin{tabular}{lll}
\hline Variables & $P$ & Correlation coefficient $(r)$ \\
\hline Age & 0.131 & 0.087 \\
Gender & 0.550 & 0.087 \\
Smoking & 0.799 & 0.085 \\
ECOG PS & 0.487 & 0.085 \\
CCI & 0.187 & 0.078 \\
Pathology type & 0.659 & 0.083 \\
EGFR mutation type & 0.593 & 0.091 \\
TNM stage & 0.522 & -0.055 \\
History of chemotherapy & 0.639 & -0.105 \\
Surgical history of lung & 0.051 & -0.21 \\
EGFR-TKI category & 0.507 & 0.062 \\
Treatment line & 0.907 & -0.032 \\
\hline
\end{tabular}

EGFR-TKI, epidermal growth factor receptor-tyrosine kinase inhibitor; ECOG PS, Eastern Cooperative Oncology Group performance status; $\mathrm{CCl}$, Charlson comorbidity index; TNM, tumor-node-metastasis.

PIL grade were significantly associated with PFS. The clinicopathological factors significantly associated with shorter PFS included squamous cell carcinoma, E21 L858R, and higher PIL grade, which were independent risk factors for disease progression. Median PFS and ORR of squamous cell carcinoma vs. adenocarcinoma were 3.0 vs. 10.0 months ( $\mathrm{HR}=4.34$; 95\% CI: $1.52-12.42, \mathrm{P}=0.006)$ and $40 \%$ vs. $74.4 \%$, respectively. Median PFS and ORR for E21 L858R vs. E19 del were 8.0 vs. 14.5 months (HR =1.57; $95 \%$ CI: $1.00-2.46, \mathrm{P}=0.049)$ and $64.5 \%$ vs. $84.5 \%$, respectively. Median PFS and ORR for PIL grade 0, 1, 2, and 3 were 12.9 months and $80.6 \%, 11.0$ months and $72.0 \%$, 10.0 months and $71.4 \%$, and 7.0 months and $52.6 \%$ $(\mathrm{P}=0.031)$, respectively. Compared to PIL grade 3, HRs for PFS of PIL grade 0, 1, and 2 were 0.40 (95\% CI: 0.21-0.76, $\mathrm{P}=0.005), 0.46$ (95\% CI: 0.23-0.93, $\mathrm{P}=0.029)$, and 0.63 (95\% CI: 0.30-1.29, P=0.20, respectively) (Table 4, Figure 2).

\section{Discussion}

EGFR-TKIs greatly improve survival and quality of life for patients with EGFR-mutant advanced NSCLC. However, acquired drug resistance impedes long-term clinical benefits and poses new challenges for treatment. Moreover, the time and patterns of recurrence among individuals are not the same. This study investigated the relationship between pre-existing PIL and EGFR-TKI resistance. The results showed that pre-existing PIL grade was an independent risk factor besides pathological type and EGFR mutation type for predicting disease progression in patients with EGFRmutant advanced NSCLC treated with EGFR-TKIs. It suggested that the presence of higher PIL grade before EGFR-TKI treatment implied shorter PFS, which might be related to early resistance to EGFR-TKIs.

Epidemiological data shows that the incidence of lung cancer in patients with pulmonary interstitial fibrosis is 3.34-7.30 times higher than in healthy people and that pulmonary interstitial fibrosis is considered an independent risk factor affecting prognosis and survival $(10-12,17,18)$. A retrospective analysis reported by Kanaji et al. showed that in advanced NSCLC patients who received first-line chemotherapy or EGFR-TKI treatment, median PFS was significantly shorter in patients with ILD (118 days) or idiopathic pulmonary fibrosis (92 days) than in those with non-ILD (196 days) (19). A previous study has shown that alveolitis was the earliest manifestation of ILD in pathology, mainly presenting as ground-glass opacity in HRCT (20). Reticulation was defined as small linear opacities that represent thickened intralobular or interlobular septa (14), while honeycomb signs represented end-stage parenchymal 
Table 4 Variables in univariate and multivariate analysis

\begin{tabular}{|c|c|c|c|c|c|}
\hline Variables & PFS (months) & $\frac{\text { Univariate }}{\mathrm{P}}$ & \multicolumn{3}{|c|}{ Multivariate } \\
\hline \multicolumn{6}{|l|}{ Pathology } \\
\hline SCC & 3.0 & 0.002 & & & 1.000 \\
\hline Ade & 10.0 & & 0.23 & $0.08-0.66$ & 0.006 \\
\hline Yes & 15.0 & 0.092 & & & 1.000 \\
\hline No & 8.0 & & 1.33 & $0.80-2.21$ & 0.27 \\
\hline \multicolumn{6}{|c|}{ EGFR mutation type } \\
\hline E21 L858R & 8.0 & 0.028 & & & 1.000 \\
\hline PIL3 & 7.0 & 0.003 & & & 1.000 \\
\hline PIL2 & 10.0 & & 0.63 & $0.30-1.29$ & 0.203 \\
\hline PIL1 & 11.0 & & 0.46 & $0.23-0.93$ & 0.029 \\
\hline PILO & 12.9 & & 0.40 & $0.21-0.76$ & 0.005 \\
\hline
\end{tabular}

PFS, progression-free survival; HR, hazard ratio; Cl, confidence intervals; SCC, squamous cell carcinoma; Ade, adenocarcinoma; PIL, pulmonary interstitial lesions.

fibrosis (16). Therefore, the present study classified PIL into grades 0 to 3 according to HRCT manifestations of the range and extent of ground-glass opacity, abnormal reticulation, and honeycomb signs in both lungs. The findings indicated that higher PIL grade was associated with shorter PFS, leading to earlier acquired EGFR-TKI resistance. Median PFS was only 7.0 months in patients with PIL grade 3 vs. 12.9 months in those with PIL grade 0 , suggesting that PIL grade may be a predictor of early resistance to EGFR-TKIs.

The mechanisms of EGFR-TKI resistance are complex. Preclinical studies have shown that EMT plays an important role in both the EGFR-TKI resistance and formation of pulmonary fibrosis $(5,6)$. TGF- $\beta$ is the main inducer in the process of EMT and is highly expressed in patients with ILD $(7,8,17)$. Persistent exposure to TGF- $\beta 1$ is necessary for tumor cells to maintain their mesenchymal phenotype and the state of EGFR-TKI resistance (21). Progression and completion of EMT processes are associated with cell invasion and drug resistance (22). EMT is a process in which epithelial cells lose their polarity and adherence, while gaining migratory ability and adopting a mesenchymal phenotype, manifesting as a loss of E-cadherin and accompanied by an increase in $\mathrm{N}$-cadherin, vimentin, and fibronectin expression (6). In vitro studies have reported that NSCLC cells resistant to gefitinib exhibit EMT features and their mesenchymal phenotype is more resistant than the epithelial phenotype (23). With these results in mind, it is possible that there might be different expression levels of TGF- $\beta$ or EMT-related proteins in pulmonary interstitial and serum of lung cancer patients with different PIL grades, which may be related to acquired resistance to EGFR-TKIs. Specific mechanisms are being studied to provide more effective predictors or monitoring indicators for early clinical evaluation of the EGFR-TKI efficacy.

EGFR-TKI-induced ILD is a rare but severe adverse effect, which may exacerbate pulmonary fibrosis, leading to a life-threatening acute event. The incidence of ILD with EGFR-TKIs is about $1.2-6.5 \%$ and the mortality rate is 13.0-40.0\% among EGFR-TKI-treated patients with ILD, mostly occurring within 4 weeks of treatment initiation (24-27). Johkoh et al. reported that ILD exacerbation occurred in $10.6 \%$ of the erlotinib-treated patients (24). In the present study, on the basis of clinical and radiological manifestations, no patients experienced acute exacerbation of pulmonary fibrosis and resulting treatment interruption 

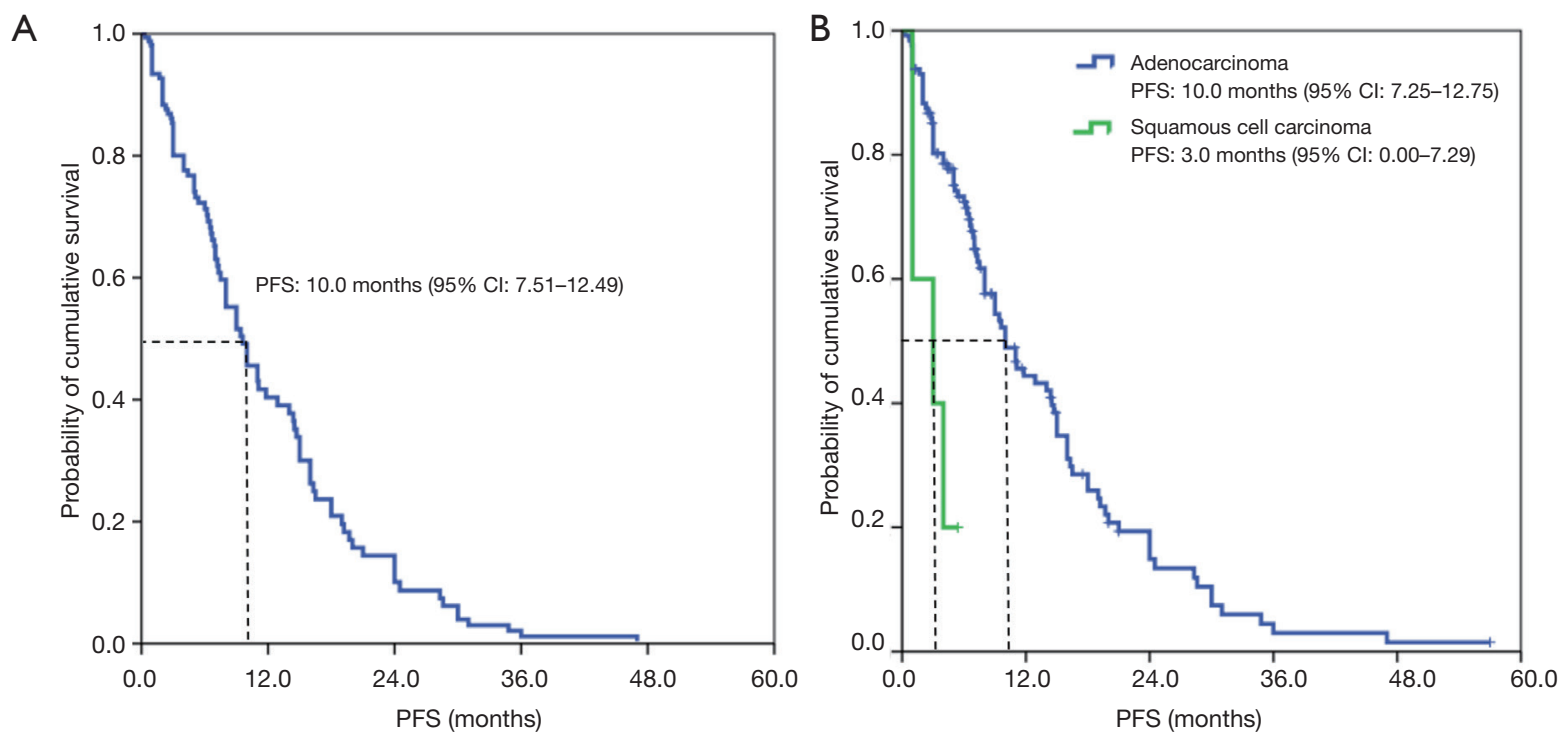

C
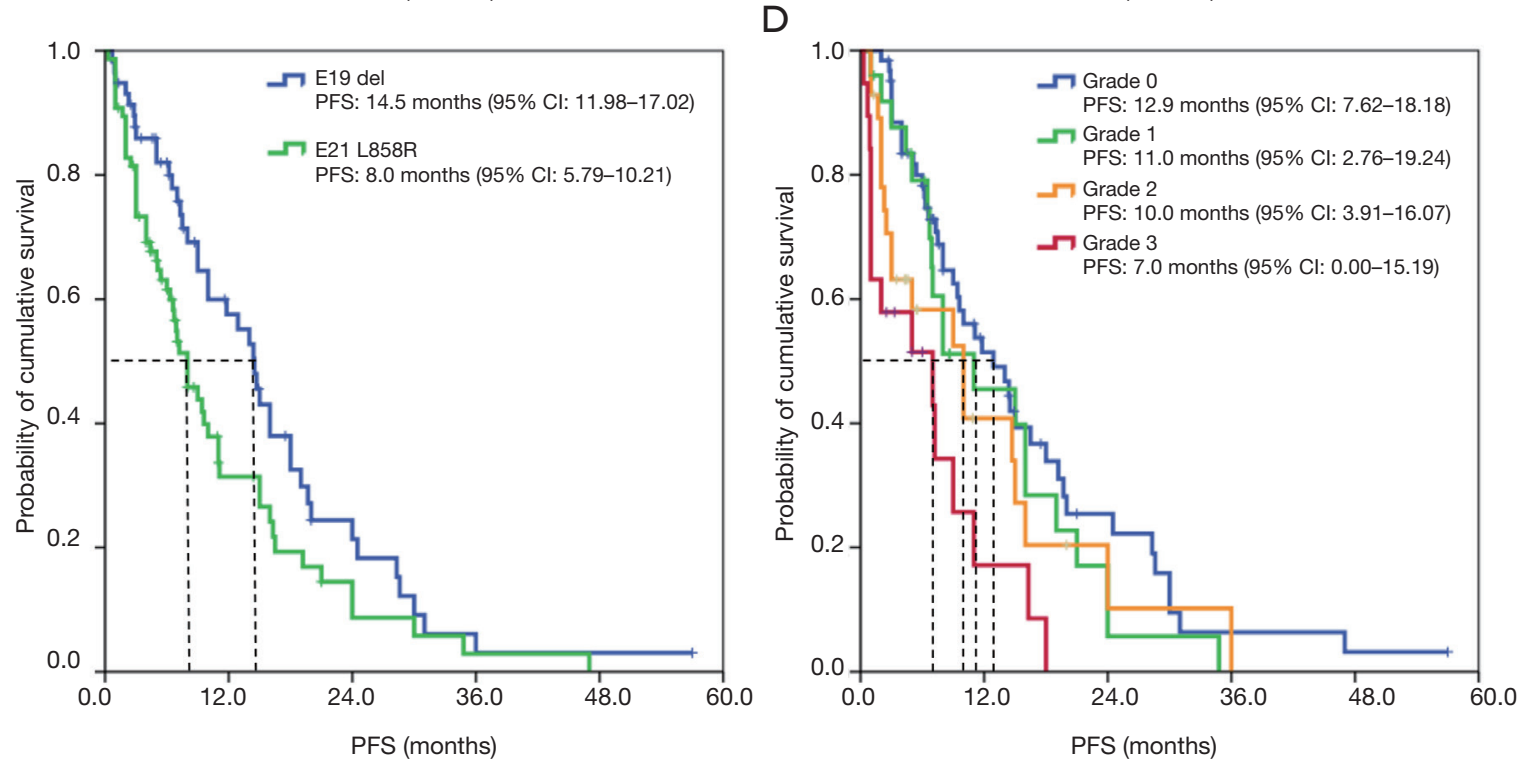

Figure 2 Curves of PFS. (A) Kaplan-Meier curves for PFS in all patients; (B) PFS in subgroups of adenocarcinoma vs. squamous cell carcinoma; (C) E19 del vs. E21 L858R; and (D) PIL grades 0, 1, 2, and 3. PFS, progression free survival; PIL, pulmonary interstitial lesions.

regardless of PIL grade. No lethal ILD outcomes were observed at the end of the last follow-up.

Furthermore, based on the conclusion between pre-existing PIL grade and resistance to EGFR-TKIs, we speculate that the combination of EGFR-TKIs and anti-pulmonary fibrosis agents might delay or prevent the onset of EGFRTKI resistance. Nintedanib is a triple angiokinase inhibitor that binds to the intracellular tyrosine kinase domain of angiogenesis-related receptors, including FGFR, PDGFR, and VEGFR. In vitro cell experiments have demonstrated that nintedanib can inhibit early TGF- $\beta 1$ signaling transduction events, downregulate expression of fibronectin, and inhibit the differentiation of myofibroblast (28). Of note, nintedanib is also able to inhibit TGF- $\beta 1$-induced phenomena of increased vimentin and decreased E-cadherin expression in A549 cells, while reversing TGF- $\beta 1$-induced EMT and resistance to gefitinib (29). However, there are few clinical trials on combination therapy with nintedanib. In the LUME-Lung 1 controlled trial, docetaxel and nintedanib group significantly improved overall survival in the second-line treatment of lung adenocarcinoma compared to docetaxel and placebo group (12.6 vs. 
10.3 months) (30). In a phase I clinical trial assessing the efficacy of afatinib combined with nintedanib, the disease control rate in 18 NSCLC patients was $83 \%$, with one complete response and three partial responses (31). Thus, whether EGFR-TKIs in combination with nintedanib can delay the emergence of acquired drug resistance in patients with pulmonary interstitial fibrosis, especially with higher PIL grades, remains to be studied further.

Generally, squamous cell lung carcinoma has a lower EGFR mutation rate (2.7-9.6\%) (32-35), so that routine testing for all squamous cell carcinoma types is not recommended by the NCCN guidelines. Nevertheless, gene testing for squamous cell carcinoma can be recommended for select patients, including light or never-smokers, patients diagnosed with small biopsy specimens, or those with mixed lung squamous cell carcinoma (36,37). Our results showed that median PFS of squamous cell carcinoma was significantly shorter than that of adenocarcinoma (3.0 vs. 10.0 months), although only five cases $(3.7 \%$ ) of lung squamous cell carcinoma were included in this study, which is not sufficient to evaluate the prognostic impact of squamous cell carcinoma. Previous studies also reported that EGFR-TKIs were less effective in EGFR-mutant nonadenocarcinoma than in adenocarcinoma. The difference in their efficacy may be related to lower levels of EGFR mutations and higher proportion of other genetic mutations in squamous cell carcinoma. PIK3CA mutation and copy number gains are more frequent in squamous cell carcinoma than in adenocarcinoma (30 vs. 3\%) (38).

This study also found that patients with E19 del had a significantly better PFS than those with E21 L858R (14.5 vs. 8.0 months), which is consistent with previous studies $(39,40)$. A meta-analysis showed that treatment with EGFR-TKIs compared to chemotherapy was significantly associated with a $63 \%$ reduction in the risk of disease progression or death. For tumors with E19 del, the benefit was greater than for tumors with E21 L858R, with unadjusted pooled HR of 0.26 and 0.45 , respectively (41).

There are some limitations to this study. First, this was a single center retrospective study with a limited number of cases that needs to be validated by well-designed prospective studies. Second, the enrolled patients received different first-generation EGFR-TKIs. Although multiple randomized controlled trials have suggested that there is a small difference in efficacy among gefitinib, erlotinib and icotinib (42), the difference in efficacy caused by the drug itself will be magnified due to the study's small sample size. Third, HRCT cannot completely distinguish atypical interstitial-like manifestations from other lung diseases (e.g., atypical adenomatous hyperplasia, adenocarcinoma in situ, and minimally invasive adenocarcinoma) and even misdiagnose physiological gravity-dependent opacification as pulmonary interstitial diseases, although dynamic followup CT can help identify these conditions to some extent. In the present study, radiologists reached a consensus on diagnosis and grade of PIL according to baseline HRCT before treatment or in combination with review of followup CT (if needed) to minimize diagnostic errors.

\section{Conclusions}

Our study suggested that pre-existing PIL might affect the efficacy of EGFR-TKIs in patients with advanced EGFR-mutant NSCLC. The higher the PIL grade, the shorter the PFS, which is related to early resistance of the EGFR-TKI treatment. Further clinical trials are needed to verify whether the combination of EGFR-TKI and anti-pulmonary fibrosis drugs can delay or prevent the occurrence of EGFR-TKI resistance.

\section{Acknowledgments}

Funding: This study was supported by grants from the National Natural Science Foundation of China (grant number 81501990).

\section{Footnote}

Conflicts of Interest: The authors have no conflicts of interest to declare.

Ethical Statement: The authors are accountable for all aspects of the work in ensuring that questions related to the accuracy or integrity of any part of the work are appropriately investigated and resolved. This study was approved by the Institutional Review Board of Shengjing Hospital of China Medical University (No. 2015PS167K). The requirement for patient consent was waived because this was a retrospective cohort study.

Open Access Statement: This is an Open Access article distributed in accordance with the Creative Commons Attribution-NonCommercial-NoDerivs 4.0 International License (CC BY-NC-ND 4.0), which permits the noncommercial replication and distribution of the article with the strict proviso that no changes or edits are made and the 
original work is properly cited (including links to both the formal publication through the relevant DOI and the license). See: https://creativecommons.org/licenses/by-nc-nd/4.0/.

\section{References}

1. Bray F, Ferlay J, Soerjomataram I, et al. Global cancer statistics 2018: GLOBOCAN estimates of incidence and mortality worldwide for 36 cancers in 185 countries. CA Cancer J Clin 2018;68:394-424.

2. Mitsudomi T, Morita S, Yatabe $Y$, et al. Gefitinib versus cisplatin plus docetaxel in patients with non-small-cell lung cancer harbouring mutations of the epidermal growth factor receptor (WJTOG3405): an open label, randomised phase 3 trial. Lancet Oncol 2010;11:121-8.

3. Zhou C, $\mathrm{Wu} \mathrm{YL}, \mathrm{Chen} \mathrm{G}$, et al. Erlotinib versus chemotherapy as first-line treatment for patients with advanced EGFR mutation-positive non-small-cell lung cancer (OPTIMAL, CTONG-0802): a multicentre, open-label, randomised, phase 3 study. Lancet Oncol 2011;12:735-42.

4. Westover D, Zugazagoitia J, Cho BC, et al. Mechanisms of acquired resistance to first- and second-generation EGFR tyrosine kinase inhibitors. Ann Oncol 2018;29:110-9.

5. Tulchinsky E, Demidov O, Kriajevska M, et al. EMT: A mechanism for escape from EGFR-targeted therapy in lung cancer. Biochim Biophys Acta Rev Cancer 2019;1871:29-39.

6. Rout-Pitt N, Farrow N, Parsons D, et al. Epithelial mesenchymal transition (EMT): a universal process in lung diseases with implications for cystic fibrosis pathophysiology. Respir Res 2018;19:136.

7. Saito A, Horie M, Micke P, et al. The Role of TGF- $\beta$ Signaling in Lung Cancer Associated with Idiopathic Pulmonary Fibrosis. Int J Mol Sci 2018;19. Pii: E3611.

8. Derynck R, Muthusamy BP, Saeteurn KY. Signaling pathway cooperation in TGF- $\beta$-induced epithelialmesenchymal transition. Curr Opin Cell Biol 2014;31:56-66.

9. Fernandez IE, Eickelberg O. The impact of TGF- $\beta$ on lung fibrosis: from targeting to biomarkers. Proc Am Thorac Soc 2012;9:111-6.

10. Naccache JM, Gibiot Q, Monnet I, et al. Lung cancer and interstitial lung disease: a literature review. J Thorac Dis 2018;10:3829-44.

11. Tzouvelekis A, Spagnolo P, Bonella F, et al. Patients with IPF and lung cancer: diagnosis and management. Lancet Respir Med 2018;6:86-8.

12. Yoon JH, Nouraie M, Chen X, et al. Characteristics of lung cancer among patients with idiopathic pulmonary fibrosis and interstitial lung disease - analysis of institutional and population data. Respir Res 2018;19:195.

13. Pipavath S, Godwin JD. Imaging of interstitial lung disease. Clin Chest Med 2004;25:455-65, v-vi.

14. Jacob J, Hansell DM. HRCT of fibrosing lung disease. Respirology 2015;20:859-72.

15. Travis WD, Costabel U, Hansell DM, et al. An Official American Thoracic Society/European Respiratory Society Statement: Update of the international multidisciplinary classification of the idiopathic interstitial pneumonias. Am J Respir Crit Care Med 2013;188:733-48.

16. Gruden JF. CT in Idiopathic Pulmonary Fibrosis: Diagnosis and Beyond. AJR Am J Roentgenol 2016;206:495-507.

17. Gu P, Luo B, Yi X, et al. The expressions and meanings of BMP-7 and TGF- $\beta$ in idiopathic pulmonary fibrosis and idiopathic nonspecific interstitial pneumonia. Zhonghua Jie He He Hu Xi Za Zhi 2014;37:664-70.

18. Lee T, Park JY, Lee HY, et al. Lung cancer in patients with idiopathic pulmonary fibrosis: clinical characteristics and impact on survival. Respir Med 2014;108:1549-55.

19. Kanaji N, Tadokoro A, Kita N, et al. Impact of idiopathic pulmonary fibrosis on advanced non-small cell lung cancer survival. J Cancer Res Clin Oncol 2016;142:1855-65.

20. Walsh SL, Hansell DM. High-resolution CT of interstitial lung disease: a continuous evolution. Semin Respir Crit Care Med 2014;35:129-44.

21. Soucheray M, Capelletti M, Pulido I, et al. Intratumoral Heterogeneity in EGFR-Mutant NSCLC Results in Divergent Resistance Mechanisms in Response to EGFR Tyrosine Kinase Inhibition. Cancer Res 2015;75:4372-83.

22. Brabletz T, Kalluri R, Nieto MA, et al. EMT in cancer. Nat Rev Cancer 2018;18:128-34.

23. Weng CH, Chen LY, Lin YC, et al. Epithelialmesenchymal transition (EMT) beyond EGFR mutations per se is a common mechanism for acquired resistance to EGFR TKI. Oncogene 2019;38:455-68.

24. Johkoh T, Sakai F, Kusumoto M, et al. Association between baseline pulmonary status and interstitial lung disease in patients with non-small-cell lung cancer treated with erlotinib--a cohort study. Clin Lung Cancer 2014; 15:448-54.

25. Skeoch S, Weatherley N, Swift AJ, et al. Drug-Induced Interstitial Lung Disease: A Systematic Review. J Clin Med 2018. doi: 10.3390/jcm7100356.

26. Beom SH, Kim DW, Sim SH, et al. Gefitinib-Induced Interstitial Lung Disease in Korean Lung Cancer Patients. Cancer Res Treat 2016;48:88-97. 
27. Saito Y, Gemma A. Current status of DILD in molecular targeted therapies. Int J Clin Oncol 2012;17:534-41.

28. Rangarajan S, Kurundkar A, Kurundkar D, et al. Novel Mechanisms for the Antifibrotic Action of Nintedanib. Am J Respir Cell Mol Biol 2016;54:51-9.

29. Nishijima N, Seike M, Soeno C, et al. miR-200/ZEB axis regulates sensitivity to nintedanib in non-small cell lung cancer cells. Int J Oncol 2016;48:937-44.

30. Reck M, Kaiser R, Mellemgaard A, et al. Docetaxel plus nintedanib versus docetaxel plus placebo in patients with previously treated non-small-cell lung cancer (LUMELung 1): a phase 3, double-blind, randomised controlled trial. Lancet Oncol 2014;15:143-55.

31. Bahleda R, Hollebecque A, Varga A, et al. Phase I study of afatinib combined with nintedanib in patients with advanced solid tumours. Br J Cancer 2015;113:1413-20.

32. Forbes SA, Bhamra G, Bamford S, et al. The Catalogue of Somatic Mutations in Cancer(COSMIC). Curr Protoc Hum Genet 2008; Chapter 10:Unit 10.11.

33. Kalemkerian GP, Narula N, Kennedy EB. Molecular testing guideline for the selection of lung cancer patients for treatment with targeted tyrosine kinase inhibitors: American Society of Clinical Oncology Endorsement Summary of the College of American Pathologists/ International Association for the Study of Lung Cancer/ Association for Molecular Pathology Clinical Practice Guideline Update. J Oncol Pract 2018;14:323-7.

34. Wang Z, Shen Z, Li Z, et al. Activation of the BMPBMPR pathway conferred resistance to EGFR-TKIs in lung squamous cell carcinoma patients with EGFR mutations. Proc Natl Acad Sci U S A 2015;112:9990-5.

35. Joshi A, Zanwar S, Noronha V, et al. EGFR mutation in squamous cell carcinoma of the lung: does it carry the same connotation as in adenocarcinomas? Onco Targets
Ther 2017;10:1859-63.

36. National Comprehensive Cancer Network. Non-Small Cell Lung Cancer (Version 3.2019). Available online: https://www.nccn.org/professionals/physician_gls/default. aspx\#nscl

37. Paik PK, Varghese AM, Sima CS, et al. Response to erlotinib in patients with EGFR mutant advanced nonsmall cell lung cancers with a squamous or squamous-like component. Mol Cancer Ther 2012;11:2535-40.

38. Shukuya T, Takahashi T, Kaira R, et al. Efficacy of gefitinib for non-adenocarcinoma non-small-cell lung cancer patients harboring epidermal growth factor receptor mutations: a pooled analysis of published reports. Cancer Sci 2011;102:1032-7.

39. Sequist LV, Yang JC, Yamamoto N, et al. Phase III study of afatinib or cisplatin plus pemetrexed in patients with metastatic lung adenocarcinoma with EGFR mutations. J Clin Oncol 2013;31:3327-34.

40. Wu YL, Zhou C, Hu CP, et al. Afatinib versus cisplatin plus gemcitabine for first-line treatment of Asian patients with advanced non-small-cell lung cancer harbouring EGFR mutations (LUX-Lung 6): an open-label, randomised phase 3 trial. Lancet Oncol 2014;15:213-22.

41. Lee CK, Wu YL, Ding PN, et al. Impact of Specific Epidermal Growth Factor Receptor (EGFR) Mutations and Clinical Characteristics on Outcomes After Treatment With EGFR Tyrosine Kinase Inhibitors Versus Chemotherapy in EGFR-Mutant Lung Cancer: A metaAnalysis. J Clin Oncol 2015;33:1958-65.

42. Liu Y, Zhang Y, Feng G, et al. Comparison of effectiveness and adverse effects of gefitinib, erlotinib and icotinib among patients with non-small cell lung cancer: A network meta-analysis. Exp Ther Med 2017;14:4017-32.
Cite this article as: Zhang XY, Cao R, Guo YJ, Zhen YH, Zheng JH, Huang LT, Zhang SL, Jing W, Sun L, Zhao JZ, Han $\mathrm{CB}, \mathrm{Ma}$ JT. Impact of pulmonary interstitial lesions on efficacy and prognosis of EGFR-TKI-treated advanced non-small cell lung cancers. J Thorac Dis 2020;12(3):839-848. doi: 10.21037/ jtd.2019.12.128 